\title{
2 Intensivtherapie bei adipösen Kindern und Jugendlichen
}

\author{
Lutz Bindl
}

\subsection{Epidemiologie und Körperzusammensetzung}

Übergewicht und Adipositas bei Kindern und Jugendlichen werden altersbezogen über den BodyMass-Index (BMI) definiert. Als übergewichtig gelten Kinder und Jugendliche mit einem BMI über der $90 \%$-Perzentile, als adipös bei einem BMI über der 97\%-Perzentile. Die Absolutwerte des BMI korrelieren nur eingeschränkt mit der Körperfettmasse, insbesondere in der Altersgruppe der über 10-Jährigen (Widhalm et al. 2001). Zu Beginn der zurückliegenden Dekade ist die Prävalenz der Adipositas bei deutschen Kindern und Jugendlichen angestiegen. Aus der nationalen CrescNet-Datenbank geht hervor, dass ca. $11 \%$ der vier bis 8-jährigen Kinder übergewichtig und 4,5\% adipös sind. Unter Kindern und Jugendlichen zwischen acht und sechzehn Jahren beträgt der Anteil 9-10\% (Blüher et al. 2011). Die Häufigkeit unter pädiatrischen Intensivpatienten ist nicht bekannt. Nach eigenen Daten aus dem Zeitraum 1991-2001 waren 6,5\% der Kinder und Jugendlichen mit Acute respiratory distress syndrome mit dem Gewicht über der $97 \%$-Perzentile.

Der Anteil der Fettmasse am Körpergewicht liegt physiologisch im zweiten Lebenshalbjahr mit etwa 25\% - im Vergleich zu 15\% beim Erwachsenen und beim Neugeborenen - besonders hoch (Rohmann u. Burmeister 1977). Dies führt oft zur fälschlichen Wahrnehmung von Säuglingen mit altersphysiologisch hoher subkutaner Fettmasse als adipös.

\subsection{Morbidität und Komorbidität adipöser Kinder und Jugendlicher}

Während technische Probleme im Umgang mit stark übergewichtigen Patienten grundsätzlich bei Jugendlichen und Erwachsenen ähnlich sind, besteht ein erheblicher Unterschied im Ausmaß der Komorbidität. Die Adipositas im Kindes- und Jugendalter stellt zwar einen Risikofaktor für kardiovaskuläre Erkrankungen dar. Klinisch relevante Gefäßveränderungen sind im Kindesalter - im Gegensatz zu subklinischen Veränderungen (Mimoun et al. 2008) in der Regel aber gering.

Andere Komplikationen der Fettleibigkeit, wie eine eingeschränkte Insulinsensitivität, prokoagulatorische Gerinnungsveränderungen oder ein vermehrter gastroösophagealer Reflux sind jedoch auch bei adipösen Kindern und Jugendlichen zu berücksichtigen.

\subsubsection{Hereditäre Erkrankungen und Syndrome mit Adipositas}

In der Minderheit der Fälle ist die Adipositas Symptom einer Grundkrankheit. Eine alimentär bedingte Adipositas findet sich gehäuft bei Kindern mit eingeschränkter Bewegungsfähigkeit, wie etwa Patienten mit Myopathien. Naturgemäß ist der Anteil chronisch kranker Patienten unter pädiatrischen Intensivpatienten über dem Populationsdurchschnitt. 
Tab. 3 Strukturelle Chromosomenanomalien mit gehäufter Adipositas

\begin{tabular}{|l|l|}
\hline numerische Aberrationen & Trisomie 21 \\
\cline { 2 - 2 } $\begin{array}{l}\text { Deletionen, } \\
\text { Mikrodeletionen und }\end{array}$ & $2 q 37$ \\
uniparentale Disomien & 4935.1 mit 5p14.3 dup \\
& (MOMES-Syndrom) \\
& $6 q 14.1-14.5$ \\
& $6 q 16.1-q 16.3$ \\
& $15 q 11$ (Prader-Willi-Syndrom) \\
& $16 p 11.2$ \\
\hline & $22 q 11$ (MURCS-Assoziation) \\
\hline Duplikationen & $19 q 12-q 13$ \\
\hline
\end{tabular}

Die Tabellen 3 und 4 enthalten Auflistungen von syndromalen Erkrankungen und Chromosomenaberrationen, die mit Adipositas vergesellschaftet sind. Als Folge von Therapiemaßnahmen findet sich die Adipositas beim iatrogenen Cushing-Syndrom durch hypothalamische Läsionen nach Bestrahlung, durch Chemotherapien, Resektion von Craniopharyngeomen (Sahakitrungruang et al. 2011) sowie durch erhöhte Substratzufuhr bei metabolischen Erkrankun- gen mit hohem Hypoglykämie-Risiko (Glykogeneosen, Nesidioblastose und andere Erkrankungen mit Hyperinsulinismus) und bei Patienten unter Valproattherapie (Verrotti et al. 2010).

Die häufigste Ursache für Adipositas im Kindesalter ist die Fehlernährung. Selten findet sich Fettleibigkeit als Folge von Therapiemaßnahmen oder metabolischen Entgleisungen.

\subsubsection{Erkrankungen und Symptome durch Adipositas}

\section{Kardiovaskuläre Risikofaktoren}

Bei adipösen Kindern sind hypertensive Blutdruckwerte gehäuft (Schiel et al. 2006). Als Ursache besteht eine Verzögerung der endothelvermittelten, postischämischen Vasodilatation (Bhattacharjee et al. 2010), in Korrelation zum Übergewicht werden vermehrt zirkulierende aktivierte Endothelzellen (Kelly et al. 2010) nachgewiesen. Stenosierende Gefäßveränderungen sind, vor allem bei gleichzeitiger familiärer Hypercholesterolämie, auch bei Kindern und Jugendlichen möglich.

Tab. 4 Hereditäre Erkrankungen mit gehäufter Adipositas

\section{mit Dysmorphien}

\begin{tabular}{|c|c|}
\hline \#209900 & $\begin{array}{l}\text { Bardet-Biedl-Syndrom (Präaxiale Oolydaktylie, Retinitis pigmentosa, Katatrakt, Retardierung, Leberfibrose, } \\
\text { Nierenanomalien) }\end{array}$ \\
\hline \#301900 & $\begin{array}{l}\text { Börjesson-Forssman-Lehman Syndrom (mentale Retardierung, Epilepsie, Hypogonadismus, Hypometabolism, } \\
\text { faziale Dysmorphien) }\end{array}$ \\
\hline \#201000 & Carpenter Syndrom (Skelettfehlbildungen, Hypogonadismus, Retardierung) \\
\hline$\# 103580$ & $\begin{array}{l}\text { Pseudohypoparathyroidismus 1a (Hypokalzämie und Hypophosphatämie, Minderwuchs, faziale } \\
\text { Dysmorphien) }\end{array}$ \\
\hline
\end{tabular}

ohne Dysmorphie

\begin{tabular}{|c|c|}
\hline$\% 184700$ & Polyzystische Ovarien-Syndrom (Adipositas, Amenorrhoe, Hirsutismus, polyzystische Ovarien) \\
\hline \#600955 & $\begin{array}{l}\text { Prohormonconvertase 1/3 - Mutationen (Schwere Adipositas, Hyperphagie, Hypoglykämie, } \\
\text { hypogonadotroper Hypogonadismus, Enteropathie) }\end{array}$ \\
\hline \#610549 & INSR-Gen-Mutationen (Typ-2-Diabetes mit Acanthosis nigricans; Adipositas nicht obligat) \\
\hline \#203800 & $\begin{array}{l}\text { Alström-Syndrom (Zapfnedystrophie, sensoneurinale Taubheit, Insulinresistenz, Nephritis, dilatative } \\
\text { Kardiomyopathie) }\end{array}$ \\
\hline
\end{tabular}

ROHHADNET (Rasch progressive Adipositas, Minderwuchs, Hypoventilation, hypothalamische, autonome Dysregulation, und Ganglioneurome 


\section{Lungenfunktion}

Von Marcus et al. (1996) werden bei 46\% der untersuchten adipösen Kinder und Jugendlichen pathologische Polysomnografie-Befunde berichtet. Schwere Formen des Schlafapnoe-Syndroms treten aber bevorzugt auf in Zusammenhang mit syndromalen, auch das zentrale Nervensystem betreffenden Grunderkrankungen wie dem Prader-Willi-Syndrom und dem Syndrom der rasch progressiven Adipositas mit Minderwuchs, Hypoventilation, hypothalamischer, autonomer Dysregulation und Ganglioneuromen (ROHHADNET).

Die Hospitalisierungsrate wegen Asthma ist bei adipösen Kindern, trotz höherer Asthmaprävalenz (Unger et al. 1990) nicht erhöht (Hom et al. 2009). Adipöse Kinder benötigen aber im Status asthmaticus bei gleicher Symptomschwere eine längere Intensivtherapie (Carroll et al. 2006).

Die Häufigkeit einer gastroösophagealen Refluxerkrankung ist in einer Studie um ein Drittel erhöht gefunden worden (Koebnick et al. 2011) und lässt somit auch ein erhöhtes Risiko für Beatmungspneumonien erwarten. Allerdings liegen hierzu keine empirischen Daten vor.

\section{Insulinresistenz, hyperglykämisch-hyperosmolares Syndrom und nonalkoholische Steatohepatitis}

Bei einer Untersuchung an 520 adipösen deutschen Kindern (mittleres Alter 14 Jahre) fanden Wabitsch et al. (2004) bei 6,7\% der Patienten Störungen der Blutzuckerkontrolle, bei 1,5\% einen manifesten Diabetes Typ 2.

Bei Jugendlichen mit insulinresistenter Hyperglykämie und Adipositas wurde mehrfach das Auftreten eines lebensbedrohlichen Bildes mit dehydratationsbedingtem hypovolämischem Schock, Hyperthermie und Rhabdomyolyse, meist ohne Ketose (hyperglykämisch-hyperosmolares Syndrom) beschrieben. Solche Patienten waren überwiegend Amerikaner afrikanischen Ursprungs. In keinem Fall war ein Hirnödem aufgetreten (Hollander et al. 2003; Carchman et al. 2005).

Transaminasenerhöhungen findet man bei 10-20\% adipöser Schulkinder, insbesondere bei einer positiven Familienanamnese für Typ-2-Diabetes und männlichem Geschlecht (Kinugasa et al. 1984; Wei et al. 2010). Diese können bereits bei Jugendlichen mit einer fortgeschrittenen Steatohepatitis einhergehen. Kinugasa et al. (1984) fanden bei adipösen Jugendlichen in fünf von elf Leberbiopsien eine Leberfibrose, in einem Fall eine Zirrhose.

\section{Traumamorbidität und -mortalität}

Brown et al. (2006) beschrieben eine höhere Morbidität und tendenziell höhere Mortalität in einer Cruppe von 54 adipösen Kindern und Jugendlichen, im Vergleich zu normalgewichtigen Altersgenossen. Wenngleich die Häufigkeit schwerer Schädel-HirnTraumata bei sonst vergleichbarer Verletzungsschwere signifikant geringer war, war die Letalität mit 15\% vs. 9\% tendenziell höher. Die Dauer der Intensivtherapie war signifikant länger, die Komplikationsrate mit 41\% vs. 22\% doppelt so hoch. Dies betraf insbesondere die Sepsisinzidenz (15\% vs. $4 \%$ ) und Wundinfektionen.

Adipöse Kinder weisen eine höhere Traumamorbidität und -mortalität auf als normalgewichtige Altersgenossen.

\subsection{Therapeutische Aspekte}

\subsubsection{Pflege}

Trotz der hohen Adipositas-Prävalenz sind extrem schwergewichtige Patienten, die mit den gängigen Ausrüstungsgegenständen nicht versorgt werden können, auf pädiatrischen Intensivstationen selten. Andererseits sind Pflegekräfte, die aus der neonatologischen Intensivmedizin kommen, an die Handhabung von Patienten mit 6o-8o kg Körpergewicht nur wenig gewöhnt. Auf entsprechend korrekte Arbeitsabläufe (auch um Erkrankungen des Bewegungsapparates bei den Pflegekräften zu vermeiden) ist deshalb zu achten.

Wichtig ist die strikte Beachtung der Massnahmen zur Dekubitus-Prophylaxe (regelmäßiges Umlagern, Luftkissen-Matratzen) und zur Prophylaxe von beatmungsassoziierten Pneumonien, die sich grundsätzlich von denen im Erwachsenenalter nicht unterscheiden.

\subsubsection{Invasive Maßnahmen und Pharmakotherapie}

\section{Prozeduren}

Übergewichtige Kinder liegen in der Regel auch in der Längenentwicklung deutlich über dem Altersdurchschnitt. Altersbasierte Faustregeln zur Auswahl und Platzierung des Tubus sind somit ungeeignet. Dies gilt auch für gewichtsbasierte Tabellen und 
die „Kleinfingerregel“. In Tabelle 5 sind längenbezogene Werte für Auswahl und Platzierungstiefe von Endotrachealtuben angegeben.

Mit dem Risiko einer schwierigen Intubation ist vor allem bei jenen Kindern und Jugendlichen zu rechnen, die eine neuromuskuläre Crunderkrankung oder Fehlbildungen im Kieferbereich haben. Da die Entwicklung der Mandibula bei mangelhaftem Kieferschluss verlangsamt sein kann („adenoide Facies“), ist der Larynx bei Patienten mit obstruktiven Schlafapnoen eventuell schwerer einzustellen. Da Kinder eine ohnehin geringere funktionelle Residualkapazität haben, stellt die Adipositas einen zusätzlichen Risikofaktor für eine schnelle Desaturation bei schwieriger Intubation dar. Tubusfehllagen nach Notfallintubation sind auskultatorisch und inspektorisch schwerer festzustellen. Bei Reanimation adipöser Kinder kommt es gehäuft zu einer inkorrekten Tubuslage (Srinavasan 2010).

Die Auskultation von Lunge und Pleura ist beeinträchtigt. Bei beatmeten Patienten scheint eine großzügigere Indikation zu Routine-Thorax-Röntgenuntersuchung gerechtfertigt. Bei der Beatmung adipöser Jugendlicher sollte auf strikte Einhaltung der Maßnahmen zur Risikoreduktion von Beatmungspneumonien geachtet werden. Die Oberkörper-Hochlagerung bietet neben dem Vorteil eines verringerten gastropharyngealen Refluxes auch eine Verlagerung des intraabdominalen Gewebes und somit eine erleichterte Zwerchfellatmung. Auch bei der Extubation sollte auf eine Hochlagerung des Patienten geachtet werden.

Gefäßzugänge: Die Anlage peripherer Venenkatheter ist erheblich schwieriger. Paravasate werden oft verspätet erkannt. Die Adipositas führt bei der Anlage arterieller Katheter in die A. radialis und zentralvenöser Katheter in die V. subclavia zu wenigen zusätzlichen Schwierigkeiten. Für die Punktion der V. jugularis ist eine 2D- bzw. Farbecho-Untersuchung des Gefäßsitus hilfreich. Die Insertionslänge des Katheters beträgt dort 10\% der Körperlänge ab Mitte des M. sternocleidomastoideus. Der Zugang über die Femoralgefäße bietet wegen der etwas erschwerten Punktion und einem häufigeren Abknicken der Zuleitungen leichte Nachteile.

Kardiopulmonaler Reanimation: Adipöse Kinder und Jugendliche haben eine signifikant geringere Überlebenschance nach kardiopulmonaler Reanimation. Srinivasan et al. (2010) berichten nach Analyse des amerikanischen Reanimationsregisters der AHA für den Zeitraum 2000-2004 über 213 adipöse Kinder mit einem medianen Alter von vier Jahren. Ihre OddsRatio betrug o, 62 für das Überleben bis zur Kranken- hausentlassung nach Reanimation im Krankenhaus. Nur 15\%, im Vergleich zu 20\% der normgewichtigen Patienten, überlebten neurologisch intakt. Im Vergleich zu normgewichtigen Reanimationspatienten litt ein geringerer Anteil unter einer kardiovaskulären Erkrankung, ein höherer Anteil an respiratorischen Störungen und malignen Erkrankungen. Als mögliche Ursachen werden die schwierigere Ventilation, eine weniger effektive Herz-Druck-Massage und eine mögliche andere Pharmakokinetik von Medikamenten, namentlich Amiodarone diskutiert.

\section{Pharmakokinetik}

Von Kindern liegen praktisch keine empirischen Daten zur Anpassung von Medikamentendosen bei Adipositas vor.

\section{Generell gilt, dass pädiatrische Medikamentendosie- rungen bereits für Substanzen mit hoher Verteilung in das Fettgewebe höher sind, da physiologischerwei- se die Fettmasse höher ist.}

Dem stehen verminderte Metabolisierungsraten für hepatisch metabolisierte Medikamente im ersten Lebenshalbjahr gegenüber. Von Erstad wurden 2004 pharmakokinetische Besonderheiten bei adipösen erwachsenen Intensivpflegepatienten zusammengefasst. Danach sollten Digoxin, Propranolol, Steroide und Theophyllin eher nach dem Idealgewicht dosiert werden, Vancomycin, Betalaktam-Antibiotika und Rocuronium eher nach dem tatsächlichen Gewicht.

Antibiotika: Empirische Daten bei Kindern fehlen. Es gibt die Empfehlung, Aminoglykoside aber auch Betalactam-Antibiotika nach dem Idealgewicht (Idealgewicht gleich Gewicht für die 50\%-Perzentile in Tabelle 5) zu dosieren (Dudley 1993).

\section{Gerinnung}

Die Adipositas ist auch im Kindesalter mit einer proinflammatorischen und prokoagulatorischen Gerinnungslage verbunden. Im Vergleich zu normgewichtigen Altersgenossen sind die Plasmaspiegel von LDL, hsCRP and TNF- $\alpha$, Fibrinogen und D-Dimeren erhöht, die von Adiponectin vermindert. Die Aktivität des vonWillebrand-faktors und von Plasminogenaktivator-Inhibitor-1 sind ebenfalls erhöht (Giordano et al. 2011). Dabei weisen PAI-1-Ag and vWF-Ag bei insulinresistenten, adipösen Jugendlichen eine drei- 
Tab. 5 Längenbezogenes Gewicht für die Intubation (als Mittelwert der geschlechtsspezifischen Perzentilen). Hinter dem Wert der 50\%-Perzentile ist das Alter, für das dieses Gewicht der 50\%-Perzentile entspricht, angegeben. http://www,cdc,gov/ growthcharts

\begin{tabular}{|c|c|c|c|c|c|}
\hline \multirow{2}{*}{$\begin{array}{l}\text { Länge } \\
\text { [cm] }\end{array}$} & \multicolumn{2}{|c|}{ Längenbezogenes Gewicht } & \multirow{2}{*}{$\begin{array}{c}\text { Tubus- } \\
\text { Durchmesser }\end{array}$} & \multicolumn{2}{|c|}{ Tubustiefe $[\mathrm{cm}]$} \\
\hline & $50 \%-P$ & 97\%-P, & & oral & nasal \\
\hline 50 & 3,5 & 4,1 & 3,5 & 9 & 11 \\
\hline 60 & 5,8 & 7,1 & 3,5 & 10 & 12 \\
\hline 70 & 8,4 & 10 & 4,0 & 10,5 & 13 \\
\hline 80 & 10,6 & 12,5 & 4,0 & 11 & 14,5 \\
\hline 90 & 13 & 15,1 & 4,5 & 12,5 & 15,5 \\
\hline 100 & 15,2 & 18,6 & 5,0 & 13,5 & 16,5 \\
\hline 110 & 18 & 26,5 & 5,5 & 14,5 & 18 \\
\hline 120 & 24,3 & 35 & 6,0 & 16 & 20 \\
\hline 130 & 27 & 40,5 & 6,5 & 16,5 & 20,5 \\
\hline 140 & 35 & 51 & 6,5 & 17,5 & 21,5 \\
\hline 150 & 41 & 65 & 7,0 & 18 & 22,5 \\
\hline
\end{tabular}

fach erhöhte Aktivität auf (Galli-Tsinopoulou et al. 2011). Eine vermehrte Plättchenaggregation findet sich bei adipösen Kindern und Jugendlichen, wenn eine Dyslipoproteinämie besteht, nicht bei normalem Serum-Lipoproteinprofil (Lohse et al. 2010).

Klinische Daten über die Frequenz von thrombotischen bzw. thromboembolischen Komplikationen bei adipösen, intensivpflegebedürftigen Kindern sind nicht publiziert. Auch eine Untersuchung an adipösen Kindern nach Polytrauma lieferte keine Daten zur Frequenz von Venenthrombosen (Brown et al. 2006).

Präliminare Daten bei Kindern deuten auf stark schwankende erforderliche Dosen von Enoxaparin bei tendenziell höherer Dosis hin (Lewis et al. 2011). Es empfiehlt sich zur Prophylaxe tiefer Venenthrombosen eine Anfangsdosierung von 2 × 0,25 mg/kg s.c. unter Kontrolle der Anti-Xa-Aktivität.

Wegen der guten Kontrollierbarkeit erscheint die Gabe von unfraktioniertem Heparin (zur Prophylaxe der tiefen Venenthrombose in der üblichen Dosis = $5.000 \mathrm{U} / \mathrm{m}^{2}$ Körperoberfläche/Tag) naheliegend.

\section{Zusammenfassung}

Die intensivmedizinische Therapie adipöser Kinder entbehrt weitgehend jeder empirischen, spezifischen Datengrundla- ge. Erwiesen ist eine höhere Komplikationsrate sowohl in akuten Notfallsituationen (Reanimation), als auch eine höhere Infektionsrate nach Traumata. Intensivpflegebedürftige adipöse Kinder leiden gehäuft unter einer chronischen Grunderkrankung, die im Wesentlichen den Verlauf und die zu erwartenden Komplikationen bestimmt. Analogieschlüsse zu Erwachsenen sind nur begrenzt möglich, da die vaskuläre Komorbidität und metabolische Veränderungen deutlich weniger fortgeschritten sind. Hinsichtlich der Anlage von Gefässzugängen und der Tubusabmessungen sollte man sich an der Körperlänge orientieren. Medikamente mit geringer Verteilung im Fettgewebe sollten nach dem Idealgewicht dosiert werden.

\section{Literatur}

Bhattacharjee R, Alotaibi WA, Kheirandish-Gozal L, Sans Capdevila 0, Gozal D (2010) Endothelial dysfunction in obese non-hypertensive children without evidence of sleep disordered breathing. BMC Pediatr 10, 8

Blüher S, Meigen C, Gausche R, Keller E, Pfäffle R, Sabin M, Werther G, Odeh R, Kiess W (2011) Age-specific stabilization in obesity prevalence in German children: A cross-sectional study from 1999 to 2008. Int | Pediatr Obes 6, e199-206

Brown CV, Neville AL, Salim A, Rhee P, Cologne K, Demetriades D (2006) The impact of obesity on severely injured children and adolescents. I Pediatr Surg 41, 88-91

Burmeister W. Romahn A (1974) Entwicklung von Depot-Fett und Zellmasse vom Neugeborenenalter bis zum Abschluss des Wachstums. Monatsschr Kinderheilkd 122, 558-559 
Carchman RM, Dechert-Zeger M, Calikoglu AS, Harris BD (2005) A new challenge in pediatric obesity: pediatric hyperglycemic hyperosmolar syndrome. Pediatr Crit Care Med 6, 20-24

Carroll CL, Bhandari A, Zucker AR, Schramm CM (2006) Childhood obesity increases duration of therapy during severe asthma exacerbations. Pediatr Crit Care Med 7, 527-531

Dudley MN (1993) Antibiotic dosing in obese children. Pediatr Infect Dis | 12, 420

Erstad BL (2004) Dosing of medicaments in morbidly obese patients in the intensive care unit setting. Intensive Care Medicine 30, 18-32

Galli-Tsinopoulou A, Kyrgios I, Maggana I, Giannopoulou EZ, Kotanidou EP, Stylianou C, Papadakis E, Korantzis I, Varlamis G (2011) Insulin resistance is associated with at least threefold increased risk for prothrombotic state in severely obese youngsters. Eur J Pediatr 170, 879-886

Giordano P, Del Vecchio GC, Cecinati V, Delvecchio M, Altomare M, De Palma F, De Mattia D, Cavallo L, Faienza MF (2011) Metabolic, inflammatory, endothelial and haemostatic markers in a group of Italian obese children and adolescents. Eur | Pediatr $170,845-850$

Hollander AS, Olney RC, Blackett PR, Marshall BA (2003) Fatal malignant hyperthermia-like syndrome with rhabdomyolysis complicating the presentation of diabetes mellitus in adolescent males.Pediatrics 111, 1447-1452

Hom J, Morley El, Sasso P, Sinert R (2009) Body mass index and pediatric asthma outcomes. Pediatr Emerg Care 25, 569-571

Kelly AS, Hebbel RP, Solovey AN, Schwarzenberg S), Metzig AM, Moran A, Sinaiko AR, Jacobs DR Jr, Steinberger I (2010) Circulating activated endothelial cells in pediatric obesity. I Pediatr 157, 547-551

Kinugasa A, Tsunamoto K, Furukawa N, Sawada T, Kusunoki T, Shimada N (1984) Fatty liver and its fibrous changes found in simple obesity of children. I Pediatr Gastroenterol Nutr 3, 408-14

Koebnick C, Getahun D, Smith N, Porter AH, Der-Sarkissian JK, Jacobsen SI (2011) Extreme childhood obesity is associated with increased risk for gastroesophageal reflux disease in a large population-based study. Int | Pediatr Obes 6, e257-263

Lewis TV, Johnson PN, Nebbia AM, Dunlap M (2011) Increased enoxaparin dosing is required for obese children. Pediatrics 127 , e787-e790

Lohse I, Schweigel J, Naeke A, Lee-Kirsch MA, Siegert G, Bergmann S, Kuhlisch E, Suttorp M, Knöfler R (2010) Platelet function in obese children and adolescents. Hämostaseologie 30 Suppl 1, S. $126-132$

Marcus CL, Curtis S, Koerner CB, Joffe A, Serwint IR, Loughlin GM (2006) Evaluation of pulmonary function and polysomnography in obese children and adolescents. Pediatr Pulmonol 21, 176183

Mimoun E, Aggoun Y, Pousset M, Dubern B, Bouglé D, Girardet IP, Basdevant A, Bonnet D, Tounian P (2008) Association of arterial stiffness and endothelial dysfunction with metabolic syndrome in obese children. J Pediatr 153, 65-70

Reilly I), Methven E, McDowell ZC, Hacking B, Alexander D, Stewart L, Kelnar CJH (2003) Health consequences of obesity. Arch Dis Child 88, 748-752

Sahakitrungruang T, Klomchan T, Supornsilchai V, Wacharasindhu S (2011) Obesity, metabolic syndrome, and insulin dynamics in children after craniopharyngioma surgery. Eur | Pediatr 170, 763-769

Schiel R, Beltschikow W, Kramer G, Stein G (2006) Overweight, obesity and elevated blood pressure in children and adolescentsEur | Med Res. 11, 97-101

Srinivasan V, Nadkarni VM, Helfaer MA, Carey SM, Berg RA for the American Heart Association National Registry of Cardiopulmonary Resuscitation Investigators (2010) Childhood obesity and survival after in-hospital pediatric cardiopulmonary resuscitation. Pediatrics 125, e481-e488

Unger R, Kreeger L, Christoffel KK (1990) Childhood obesity: medical and familial correlates. Clin Pediatr 29, 368-373

Verrotti A, Manco R, Agostinelli S, Coppola G, Chiarelli F (2010) The metabolic syndrome in overweight epileptic patients treated with valproic acid. Epilepsia 51, 268-273

Wabitsch M, Hauner H, Hertrampf M, Muche R, Hay B, Mayer H, Kratzer W, Debatin KM, Heinze E (2004) Type II diabetes mellitus and impaired glucose regulation in Caucasian children and adolescents with obesity living in Germany. Int I Obes Relat Metab Disord 28, 307-313

Wei C, Ford A, Hunt L, Crowne EC, Shield JP (2011) Abnormal liver function in children with metabolic syndrome from a UK-based obesity clinic. Arch Dis Child 96, 1003-1007

Widhalm K, Schönegger K, Huemer C, Auterith A (2001) Does the $B M I$ reflect body fat in obese children and adolescents? A study using the TOBEC method. Int I Obes Relat Metab Disord 25, 279285

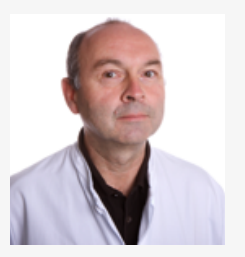

PD Dr. Lutz Bindl

Seit 1991 Arzt für Kinder-und Jugendmedizin mit dem Schwerpunkt Neonatologie und Spezialisierung in Kindergastroenterologie und pädiatrischer Intensivmedizin. Die Habilitation erfolgte zum Thema „Akutes Lungenversagen (ARDS) bei Kindern“. Oberärztlicher Leiter der pädiatrischen Intensivstationen an den Universitätsklinika Bonn und Aachen, seit 2006 am Klinikum in Luxemburg (CHL, Centre Hospitalier de Luxembourg) beschäftigt. 